\title{
The Role of Cardiovascular Risk Factors and Stroke in Familial Alzheimer Disease
}

\author{
Giuseppe Tosto, MD, PhD; Thomas D. Bird, MD; David A. Bennett, MD; Bradley F. Boeve, MD; Adam M. Brickman, PhD; Carlos Cruchaga, PhD; \\ Kelley Faber, MS; Tatiana M. Foroud, PhD; Martin Farlow, MD; Alison M. Goate, DPhil; Neill R. Graff-Radford, MD; Rafael Lantigua, MD; \\ Jennifer Manly, PhD; Ruth Ottman, PhD; Roger Rosenberg, MD; Daniel J. Schaid, PhD; Nicole Schupf, PhD; Yaakov Stern, PhD; \\ Robert A. Sweet, MD; Richard Mayeux, MD, MSc; for the National Institute on Aging Late-Onset Alzheimer Disease/National Cell \\ Repository for Alzheimer Disease (NIA-LOAD/NCRAD) Family Study Group
}

IMPORTANCE The contribution of cardiovascular disease (CV) and cerebrovascular disease to the risk for late-onset Alzheimer disease (LOAD) has been long debated. Investigations have shown that antecedent CV risk factors increase the risk for LOAD, although other investigations have failed to validate this association.

OBJECTIVE To study the contribution of CV risk factors (type 2 diabetes, hypertension, and heart disease) and the history of stroke to LOAD in a data set of large families multiply affected by LOAD.

DESIGN, SETTING, AND PARTICIPANTS The National Institute on Aging Late-Onset Alzheimer Disease/National Cell Repository for Alzheimer Disease family study (hereinafter referred to as NIA-LOAD study) is a longitudinal study of families with multiple members affected with LOAD. A multiethnic community-based longitudinal study (Washington Heights-Inwood Columbia Aging Project [WHICAP]) was used to replicate findings. The 6553 participants in the NIA-LOAD study were recruited from 23 US Alzheimer disease centers with ongoing data collection since 2003; the 5972 WHICAP participants were recruited at Columbia University with ongoing data collection since 1992. Data analysis was performed from 2003 to 2015.

MAIN OUTCOMES AND MEASURES Generalized mixed logistic regression models tested the association of CV risk factors (primary association) with LOAD. History of stroke was used for the secondary association. A secondary model adjusted for the presence of an apolipoprotein $E(A P O E) \varepsilon 4$ allele. A genetic risk score, based on common variants associated with LOAD, was used to account for LOAD genetic risk beyond the $A P O E \varepsilon 4$ effect. Mediation analyses evaluated stroke as a mediating factor between the primary association and LOAD.

RESULTS A total of 6553 NIA-LOAD participants were included in the analyses (4044 women [61.7\%]; 2509 men [38.3\%]; mean [SD] age, 77.0 [9] years), with 5972 individuals from the WHICAP study included in the replication sample (4072 women [68.2\%]; 1900 men [31.8\%]; mean [SD] age, 76.5 [7.0] years). Hypertension was associated with decreased LOAD risk (odds ratio [OR], 0.63; $95 \% \mathrm{Cl}, 0.55-0.72$ ); type 2 diabetes and heart disease were not. History of stroke conferred greater than 2-fold increased risk for LOAD (OR, 2.23; 95\% Cl, 1.75-2.83). Adjustment for $A P O E \varepsilon 4$ did not alter results. The genetic risk score was associated with LOAD (OR, 2.85; $95 \% \mathrm{Cl}$, 2.05-3.97) but did not change the independent association of LOAD with hypertension or stroke. In the WHICAP sample, hypertension was not associated with LOAD (OR, 0.99; 95\% Cl, 0.88-1.11), whereas history of stroke increased the risk for LOAD (OR, 1.96; $95 \% \mathrm{Cl}, 1.56-2.46)$. The effect of hypertension on LOAD risk was also mediated by stroke in the NIA-LOAD and the WHICAP samples.

CONCLUSIONS AND RELEVANCE In familial and sporadic LOAD, a history of stroke was significantly associated with increased disease risk and mediated the association between selected CV risk factors and LOAD, which appears to be independent of the LOAD-related genetic background.

JAMA Neurol. 2016;73(10):1231-1237. doi:10.1001/jamaneurol.2016.2539

Published online August 15, 2016.
Supplemental content at jamaneurology.com
Author Affiliations: Author affiliations are listed at the end of this article.

Group Information: A complete list of investigators of the National Institute on Aging Late-Onset Alzheimer Disease/National Cell Repository for Alzheimer Disease (NIA-LOAD/NCRAD) Family Study Group appears at the end of this article.

Corresponding Author: Richard Mayeux MD, MSc, Taub Institute for Research on Alzheimer's Disease, The Aging Brain and the Gertrude $\mathrm{H}$. Sergievsky Center, Columbia University College of Physicians and Surgeons, 630 W 168th St, Building P\&S, Box 16, New York, NY 10032 (rpm2@cumc.columbia.edu). 
ate-onset Alzheimer disease (LOAD) can be considered one of the largest unmet medical needs in the world, and available treatments show only small effects in slowing the disease progression. ${ }^{1}$ Prevention is of primary importance, and epidemiologic studies have aimed to identify modifiable risk factors. Numerous investigations have shown that LOAD risk is increased in the presence of antecedent cardiovascular (CV) risk factors, such as history of type 2 diabetes (T2D), ${ }^{2,3}$ hypertension, smoking, ${ }^{4}$ lipid disorders, ${ }^{5}$ and cerebrovascular factors, alone or in aggregate. ${ }^{6}$ Some studies suggest that CV disease (CVD) may affect dementia through multiple mechanisms, including reduction in cerebral blood flow ${ }^{7}$ and breakdown of the blood-brain barrier. ${ }^{8}$

Most of these findings come from cross-sectional clinical studies, whereas observational and neuropathologic investigations have not revealed consistent associations between T2D, hypertension, or intracranial atherosclerosis and incident LOAD or neuropathologic hallmarks (plaques or tangles). ${ }^{9}$ Thus, causal associations and the timing of CVD and cerebrovascular events with regard to LOAD remain unknown.

The largest attempt to study the contribution of CVD antecedents in LOAD was performed by the National Alzheimer Coordinating Center, capitalizing on a large collection of brain autopsies. ${ }^{10}$ First, brains with LOAD were found to harbor significantly more vascular abnormalities compared with other common neurodegenerative diseases (consistent with previous reports ${ }^{11}$ ). Second, the brains of people with comorbid LOAD and CVD showed a lower burden of amyloid and tau abnormalities. These observations suggest that CVD may lower the threshold for detection of clinical dementia. ${ }^{12}$ Studies also reported that concomitant CV abnormalities in patients with LOAD correlate with a faster decline in cognitive performance. However, other observations suggested that $\mathrm{AD}$ pathologic changes may be solely responsible for dementia manifestations, and neither macroscopic nor microscopic infarcts influence the overall state and progression of cognitive decline..$^{13}$

The aim of this study was to assess the association among CV risk factors, a history of stroke, and LOAD among families with multiple members affected by the disease. Such familial aggregation of $\mathrm{LOAD}$ provides a unique scenario for exploring the association. A family history of LOAD is, after age, the most important predictor for developing LOAD among unaffected individuals. ${ }^{14}$ Densely affected families with LOAD have been successfully used in genetic studies because they are expected to carry higher frequencies of risk alleles. Probands with a family history of LOAD more frequently have well-established disease hallmarks (ie, a higher frequency of the apolipoprotein $\mathrm{E}$ [APOE] $\varepsilon 4$ allele or lower cerebrospinal fluid levels of $\beta$-amyloid 42) compared with individuals without a family history. Taken together, these features provide a powerful scenario in which to investigate the interaction between complex diseases and multiple risk factors.

\section{Methods}

Patients and Setting

The National Institute on Aging Late-Onset Alzheimer Disease/ National Cell Repository for Alzheimer Disease family study

\section{Key Points}

Question What is the contribution of cardiovascular risk factors and stroke to the risk for late-onset Alzheimer disease (LOAD)?

Findings In this assessment of data from 2 longitudinal studies, in families multiply affected with LOAD, hypertension was associated with decreased disease risk, whereas a history of stroke was associated with a 2 -fold increased risk for developing the disease in familial and sporadic forms.

Meaning A history of stroke increases the risk for LOAD and by mediating the effect of cardiovascular risk factors.

(hereinafter referred to as NIA-LOAD study) is a collaboration among 23 participating AD centers in the United States with recruitment criteria that included families with multiple members affected by LOAD who could provide clinical information and a biological sample for DNA extraction. The proband was required to have a diagnosis of definite or probable LOAD with onset after 60 years of age and a full sibling with definite, probable, or possible LOAD with onset after 60 years of age. A third biologically related family member was required who could have been a first-, second-, or third-degree relative of the affected sibling pair and who was 60 years or older if unaffected or 50 years or older if diagnosed as having LOAD or mild cognitive impairment. Clinical diagnosis of probable or possible $\mathrm{AD}$ was made according to the criteria of the National Institute of Neurological and Communicative Diseases and Stroke and the Alzheimer's Disease and Related Disorders Association (NINCDS-ADRDA). ${ }^{15}$ Unaffected persons were required to have documented results of cognitive testing and clinical examination verifying the clinical designation. For those participants with advanced disease or who were living in a remote location and could not complete a detailed inperson evaluation, the site investigator conducted a detailed review of medical records to document the presence or absence of LOAD. Collection of data for the NIA-LOAD study has been ongoing since 2003. Each AD center received approval by their institutional review board. All participants were recruited after providing written informed consent.

The Washington Heights-Inwood Columbia Aging Project (WHICAP) is a prospective, population-based study of aging and dementia in Medicare recipients 65 years and older residing in northern Manhattan (Washington Heights, Hamilton Heights, and Inwood). The study has been described in detail elsewhere. ${ }^{16}$ Collection of data for the WHICAP has been ongoing since 1992; these data were used as a replication sample for the present study. The WHICAP was approved by the institutional review board of Columbia University, New York City, and all participants provided written informed consent.

For this study, family members from the NIA-LOAD study and other participants were required to (1) be 60 years or older at the time of enrollment; (2) have a diagnosis of probable or possible LOAD according to NINCDS-ADRDA criteria; (3) have an assessment of all CV risk factors listed as study variables (see below); and (4) have available pedigree information. 


\section{Outcome}

Primary outcomes of the study were clinically diagnosed probable or possible LOAD based on NINDS-ADRDA criteria as previously reported. ${ }^{15} \mathrm{~A}$ list of $\mathrm{AD}$ centers and their contribution to the study can be found in eFigure 1 in the Supplement.

\section{Study Variables}

The CV risk factors included as primary study variables were hypertension, T2D, and heart disease. These conditions were assessed at the time of enrollment in the study as binary variables (ie, present or absent). Hypertension was defined according to National Heart Lung and Blood Institute criteria ${ }^{17}$ as systolic blood pressure of $140 \mathrm{~mm} \mathrm{Hg}$ or higher, diastolic blood pressure of $90 \mathrm{~mm} \mathrm{Hg}$ or higher, or use of antihypertensives. Type 2 diabetes and corresponding disease-specific medications were ascertained by self-report and medication review. Type 2 diabetes was defined by a history of T2D or high blood glucose levels or treatment of T2D or high blood glucose levels reported by the participant. Heart disease included a history of myocardial infarction, congestive heart failure, or any other type of heart disease reported by the participant. As a secondary predictor we considered a history of stroke, defined according to the World Health Organization collaborative study ${ }^{18}$ (rapidly developing clinical symptoms and/or signs of focal and at times global loss of cerebral function-applied to patients in deep coma and to those with subarachnoid hemorrhage-with symptoms lasting $>24$ hours or leading to death, and with no apparent cause other than vascular). Transient ischemic attacks were excluded from this definition.

\section{Genetic Risk for LOAD}

We computed a genetic risk score (GRS) for LOAD based on the genome-wide significant single-nucleotide polymorphisms (SNPs) other than APOE \&4 (NCBI Entrez Gene 348), published from a large genome-wide association meta-analysis of LOAD. ${ }^{19}$ Genotypes for these SNPs were obtained for those NIALOAD individuals with available genome-wide association study data (a full description of genotype array, quality control, and imputation methods is found in Wijsman et $\mathrm{al}^{2 \mathrm{O}}$ ). For each individual, we multiplied the imputed probability of the risk allele (ranging from $\mathrm{O}$ to 2 ) by the reported corresponding $\beta$ coefficient, derived from the cited meta-analysis, and ultimately summed the product of each locus. In this way, we weighted each SNP by the expected effects on LOAD. Full description of SNPs and odds ratio (OR) used to derive the GRS is given in the eTable 1 in the Supplement.

\section{Statistical Analysis}

Data were analyzed from 2003 to 2015. The association of each CV risk factor with LOAD was studied in separate age-, sex-, and educational level-adjusted generalized mixed logistic regression models that included the different $\mathrm{AD}$ centers and the family as random effects to adjust for possible center variability and intercorrelation within each family cluster (model 1). Secondary models included the presence of at least $1 A P O E \varepsilon 4$ allele as an additional covariate (model 2). We applied the Nelder-Mead method of optimization to the mixed models. ${ }^{21}$

\begin{tabular}{|c|c|c|}
\hline \multirow[b]{2}{*}{ Characteristic } & \multicolumn{2}{|c|}{ No. (\%) of Participants ${ }^{\mathrm{a}}$} \\
\hline & $\begin{array}{l}\text { Clinical Sample } \\
(\mathrm{n}=6553)\end{array}$ & $\begin{array}{l}\text { Genetic Sample } \\
(n=2567)\end{array}$ \\
\hline No. of participating study centers & 23 & 23 \\
\hline Female sex & $4044(61.7)$ & $1543(60.1)$ \\
\hline Age, mean (SD), y & $77.0(9.0)$ & $76.6(9.0)$ \\
\hline Educational level, mean (SD), y & $11.6(5.0)$ & $13.8(3.0)$ \\
\hline APOE \&4 allele & $3059(52.6)$ & $1336(52.0)$ \\
\hline LOAD & $3468(52.9)$ & $1243(48.4)$ \\
\hline Hypertension & $3086(52.6)$ & $1209(47.1)$ \\
\hline T2D & $826(14.1)$ & $278(10.8)$ \\
\hline Heart disease & $1280(22.1)$ & $621(24.2)$ \\
\hline Stroke & $566(9.6)$ & $220(8.6)$ \\
\hline
\end{tabular}

Abbreviations: $A P O E$, apolipoprotein $\mathrm{E}$; LOAD, late-onset Alzheimer disease; NIA-LOAD, National Institute on Aging Late-Onset Alzheimer Disease family study; T2D, type 2 diabetes.

a Percentages have been weighted.

The same statistical approach was performed for the history of stroke. First, it was treated an independent variable for LOAD (ie, regardless of the presence or absence of hypertension, T2D, and heart disease) by using the generalized mixed logistic regression approach described above. Second, we tested the potential mediating effect of stroke on the association between the primary CV risk factors and LOAD. A mediator affects the influence of a given independent variable (in our case, hypertension, T2D, and heart diseases) on a given dependent variable (in our case, LOAD). ${ }^{22}$ We used the Sobel test ${ }^{23}$ with the $\beta$ coefficients and SEs derived from the generalized mixed models. The test reports the $P$ values from the unit normal distribution under the assumption of a 2-tailed $z$ test of the hypothesis that the mediated effect equals zero in the population. The analyses for the WHICAP sample, which consists of unrelated individuals recruited by a single study center, used a standard logistic regression approach.

We also explored the association between CVD risk factors and the genetic risk profile associated with LOAD by running generalized logistic regression mixed models where the APOE $\varepsilon 4$ allele and the GRS were entered separately as main variables and each CV risk factor was entered as a designated outcome. Additional generalized mixed-effects models (model 3) tested separately those CV risk factors found to be significant in models 1 and 2, with the GRS as an additional covariate, to account for the genetic risk profile beyond the APOE $\varepsilon 4$ allele. All analyses were conducted in R software (version 3.0; http://www.cran.org).

\section{Results}

\section{Demographics}

Clinical characteristics for the NIA-LOAD sample are reported in Table 1 and eFigure 1 in the Supplement. A total of 6553 participants were included in the analyses ( 4044 women [61.7\%]; 2509 men [38.3\%]; mean [SD] age, 77.0 [9.0] years), 
Table 2. Generalized Mixed-Effects Model Using the Vascular Risk Factors and Main Variables With LOAD as the Selected Outcome

\begin{tabular}{lllc}
\hline Variables & No. of Participants & OR $(95 \% \mathrm{Cl})$ & $P_{\text {Value }}$ \\
\hline Model $1^{\text {b }}$ & & & $<.001$ \\
\hline Hypertension & 5627 & $0.63(0.55-0.72)$ & .74 \\
\hline T2D & 5621 & $0.87(0.80-1.17)$ & .09 \\
\hline Heart disease & 5544 & $0.87(0.74-1.02)$ & $<.001$ \\
\hline Stroke & 5641 & $2.23(1.75-2.83)$ & $<.001$ \\
\hline \begin{tabular}{l} 
Model 2 \\
\hline Hypertension
\end{tabular} & 5308 & $0.63(0.54-0.72)$ & .83 \\
\hline T2D & 5303 & $0.98(0.80-1.20)$ & .08 \\
\hline $\begin{array}{l}\text { Heart disease } \\
\text { Stroke }\end{array}$ & 5228 & $0.85(0.71-1.02)$ & $<.001$ \\
\hline Model 3 & 5320 & $2.29(1.75-2.99)$ & $<.001$ \\
\hline Hypertension & 2442 & & $<.001$ \\
\hline Stroke & 2484 & $0.67(0.55-0.84)$ & \\
\hline
\end{tabular}

Abbreviations: LOAD, late-onset Alzheimer disease; OR, odds ratio; T2D, type 2 diabetes.

a Signficance was tested after multiple testing adjustment.

b Sex, age at baseline, and educational level were entered as covariates.

${ }^{c}$ Additionally adjusted for the apolipoprotein $\mathrm{E} \varepsilon 4$ allele.

${ }^{\mathrm{d}}$ Additionally adjusted for the genetic risk score. of whom 3468 (52.9\%) were diagnosed as having LOAD. In addition, 2567 participants (39.2\%) also had genome-wide association study data available. A total of 5972 participants from the WHICAP study were included in the replication sample (4072 women [68.2\%]; 1900 men [31.8\%]; mean [SD] age, 76.5 [7.0] years), of whom 2684 (45.2\%) were diagnosed as having LOAD. Full clinical characteristics for the WHICAP study are reported in eTable 2 in the Supplement.

\section{Risk Factors and LOAD}

In the generalized mixed logistic regression model (model 1), hypertension was associated with a lower risk for LOAD (OR, 0.63; 95\% CI, 0.55-0.72), whereas neither T2D nor heart disease was associated with LOAD. In contrast, a history of stroke conferred a 2-fold increased risk for LOAD (OR, 2.23; 95\% CI, 1.75-2.83). A subsequent model adjusting for the APOE $\varepsilon 4$ allele (model 2) did not change these findings (Table 2).

For the WHICAP sample, hypertension was not associated with the risk for LOAD (OR, 0.99; 95\% CI, 0.88-1.11), nor was T2D (OR, 1.09; 95\% CI, 0.95-1.25) or heart disease (OR, 1.09; 95\% CI, 0.95-1.25). In contrast, a history of stroke again conferred approximately a 2 -fold increased risk for $\operatorname{LOAD}(\mathrm{OR}, 1.96$; 95\% CI, 1.56-2.46).

\section{GRS, CV Risk Factors, and LOAD}

The frequency of the $A P O E \varepsilon 4$ allele is reported in Table 1 , and the distribution of the GRS is shown in eFigure 2 in the Supplement. A strong association with LOAD was found for the APOE $\varepsilon 4$ allele (OR, 4.47; 95\% CI, 3.86-5.17) and the GRS (OR, 2.85; 95\% CI, 2.05-3.97).

Hypertension was inversely associated with the presence of the APOE $\varepsilon 4$ allele (OR, 0.76; 95\% CI, 0.64-0.90) and the GRS (OR, 0.75; 95\% CI, 0.57-0.98); no associations were found with the other CV risk factors or a history of stroke. The association among hypertension, a history of stroke (ie, variables that were significant in models 1 and 2), and LOAD was tested again with GRS as an additional covariate; their associations with LOAD were further confirmed (model 3 in Table 2).

\section{Mediation Analyses}

As expected, a history of stroke was significantly associated with hypertension (OR, 2.08; 95\% CI, 1.69-2.57), T2D (OR, 2.06; 95\% CI, 1.62-2.61), and heart disease (OR, 2.06; 95\% CI, 1.662.54). Stroke was significantly associated with the development of LOAD. The Sobel test showed that a history of stroke was a significant mediator of hypertension $\left(P=6.7 \times 10^{-6}\right)$, T2D $\left(P=2.5 \times 10^{-5}\right)$, and heart disease $\left(P=4.0 \times 10^{-5}\right)$ in determining LOAD risk (eFigure 3 in the Supplement). In the WHICAP sample, the Sobel test also confirmed that a history of stroke was a significant mediator of hypertension $\left(P=1.3 \times 10^{-6}\right)$, T2D $\left(P=2.3 \times 10^{-4}\right)$, and heart disease $\left(P=5.8 \times 10^{-7}\right)$ in determining LOAD risk.

\section{Discussion}

The contribution of CV risk factors to the risk for developing dementia, and more specifically LOAD, remains controversial. Until recently, CV burden has been agreed to increase the risk for LOAD. More recently, leveraging on large sample sizes and accessible clinical and genetic data has shown that the association between CVD and LOAD is clearly more complex than expected.

We found an inverse association between hypertension and the risk for LOAD. This association was also observed using LOAD as defined by its genetic risk profile. The frequency of the APOE $\varepsilon 4$ allele and mean GRS were also lower in participants with a history of hypertension, whereas no association was found for the other CV risk factors. This inverse association has already been observed for $A P O E,{ }^{24}$ whereas, to our knowledge, the data for the GRS have never been reported. This finding is, to some extent, consistent with that recently reported in a large study on non-Hispanic white patients ${ }^{25}$ among whom genetically predicted higher systolic blood pressure was associated with a lower LOAD risk. That investigation used a mendelian randomization approach in which hypertension and other CVD risk factors were defined by associated SNPs from published large 
genome-wide association studies. In addition, none of the other established CVD risk factors, such as lipid levels, body mass index, and T2D, was significantly associated with LOAD.

The potential explanations for these results are multiple. First, as suggested by other studies, high blood pressure in late life may protect against LOAD. ${ }^{26,27}$ Second, a protective effect of antihypertensive treatment might explain the inverse association with hypertension. Extensive reporting shows that many compounds have beneficial effects toward the development of dementia, and cognitive impairment in general, including diuretics, ${ }^{28} \beta$-blockers, ${ }^{29}$ calcium channel blockers, ${ }^{30}$ angiotensin II receptor blockers, ${ }^{31}$ and angiotensinconverting enzyme inhibitors. ${ }^{32,33}$

More important, this finding was not replicated in the WHICAP sample, although the study design for the WHICAP relies on community-based enrollment of older adults who were dementia free at baseline and derived from 3 ethnic groups. Therefore, inclusion criteria for the 2 studies are deeply different.

Among all conditions, a history of stroke was found to be associated with a LOAD diagnosis. This finding is consistent with prior observations ${ }^{12}$ in which hazards ratios for LOAD were increased among individuals with a history of stroke, compared with those without stroke. In the present study, stroke was weakly associated with LOAD in the absence of other CV risk factors; on the contrary, risk increased with the addition of hypertension, T2D, or heart disease. This finding was replicated in the WHICAP sample. These independent conclusions suggest a mediation effect that we consistently demonstrate in our analyses.

Attesting a causal association is difficult, especially without a prospective longitudinal assessment; nevertheless, several recent observations support the hypothesis that stroke precedes the diagnosis of LOAD and not vice versa. ${ }^{34}$ Our results in the subsample with available GRS also suggest that stroke is not correlated with a specific genetic background but independently increases the risk for LOAD. Although the indirect pathway follows a risk series of associations (hypertension leading to increased stroke risk leading to increased LOAD risk), the direct pathway that shows the opposite direction (hypertension leading to decreased LOAD risk) is still plausible. This latter pathway is commonly referred to in the literature as inconsistent mediation. The assumption of mediation analysis is that the direct and indirect pathways operate simultaneously on the outcome (Y). Thus, although a change in the predictor (X [hypertension in this case]) might increase Y (LOAD in this case) through the mediator ( $M$ [stroke in this case]), the change in $\mathrm{X}$ could still yield a net decrease in $\mathrm{Y}$, if the direct effect of $\mathrm{X}$ is (1) in the opposite direction and (2) large enough to compensate for the indirect effect ${ }^{35}$ (eFigure 3 in the Supplement). Thus, hypertension could be associated with a lower risk for LOAD, but, where hypertension leads to a stroke, a higher risk for LOAD is inevitable.

These results imply that CV risk factors are associated with LOAD, although the direction of this effect might vary. This association might also depend on several conditions that we could not completely disentangle (ie, age at onset of risk fac- tors, coexistence of multiple CV risk factors, and ultimately their associated treatments), and further investigations are needed. In contrast, stroke emerges with a solid association with increased LOAD risk and a mediator of the primary CVD risk factors. Thus, regardless of the CVD risk factor profile, in the presence of a cerebrovascular event, the risk for LOAD is increased.

Consistent with this hypothesis, Tosto et $\mathrm{al}^{36}$ previously demonstrated that white matter hyperintensities, a wellestablished marker of cerebrovascular changes, are significantly associated with incident LOAD and faster progression of disease. In addition, another study by Tosto et $\mathrm{al}^{37}$ demonstrated that white matter hyperintensities promote tau accumulation and not vice versa, further proving that cerebrovascular changes precede LOAD-related neurodegenerative processes. The protective effect of hypertension on LOAD and its association with stroke, which in turn increases the risk for LOAD, depicts a complex scenario of multiple intercorrelated causal effects that cannot be reduced to a single variableoutcome association.

Another major conclusion of the present study is the lack of association between T2D and LOAD. Such an association appears to be somewhat more consistent in the literature, with rate ratios ranging from 1.3 to 4.4 in several large cohort studies. ${ }^{38}$ Nevertheless, this association is again questioned when more proximal LOAD endophenotypes or neuropathologic indexes are used effectively. Most of the studies providing longitudinal clinical follow-up paired with neuropathologic assessment concluded that, although T2D increases the risk for dementia significantly during the life course, at autopsy this condition is associated with vascular abnormalities but not with plaques or tangles. ${ }^{39-41}$ Type 2 diabetes does not show an association with carbon 11-labeled Pittsburgh Compound B or cerebrospinal fluid levels of $\beta$-amyloid 42, whereas increases in cerebrospinal fluid levels of tau, a nonspecific marker of neurodegeneration, are associated with T2D. ${ }^{42}$ This association suggests that T2D as an independent factor of degeneration with little effect on LOAD-related mechanisms and pathways. The lack of significant results in our analyses is consistent with these mechanistic models; the mediation model further proves that only through cerebrovascular disease is a significant effect of T2D on the LOAD risk found.

This study has several limitations. First, important confounders (ie, smoking, physical activity, and body mass index, to list a few) are currently missing in the proposed analyses because they were not part of the shared set of variables among the participating study centers, or because they showed high rates of missing data. Second, predictors such as heart disease or medications rely on self-reported information and, thus, are pruned to obvious biases. Third, history of stoke did not account for multiple stroke events, and the lag between the clinical event and the study enrollment was not considered. Ultimately, because of the nature of the collected data (a large complex family pedigree with only cross-sectional data), we did not provide an estimate of the mediation effect, but only reported if this was statistically significant between study variables and the outcome. 


\section{Conclusions}

The present study underlines the importance of vascular risk factors in the context of familiar LOAD. By leveraging on genetically loaded multiplex families, we further demonstrated the importance of the vascular component in high- risk individuals. This result suggests (1) the critical importance of interventions targeting modifiable risk factors in LOAD and (2) the complex relationships between hypertension (and potentially the beneficial use of antihypertensive treatments) and LOAD as previously showed by other investigations. Further studies with longitudinal assessment and a larger set of variables (eg, different classes of treatments) are currently needed.

\section{ARTICLE INFORMATION}

Accepted for Publication: May 26, 2016.

Published Online: August 15, 2016. doi:10.1001/jamaneurol.2016.2539.

Author Affiliations: Taub Institute for Research on Alzheimer's Disease, The Aging Brain and the Gertrude H. Sergievsky Center, Columbia University College of Physicians and Surgeons, New York, New York (Tosto, Brickman, Manly, Ottman, Schupf, Stern, Mayeux); Department of Neurology, Columbia University College of Physicians and Surgeons, New York, New York (Tosto, Brickman, Manly, Schupf, Stern, Mayeux); New York Presbyterian Hospital in New York City (Tosto, Brickman, Manly, Schupf, Stern, Mayeux); Department of Neurology, University of Washington, Seattle (Bird); Department of Medicine, University of Washington, Seattle (Bird); Rush Alzheimer's Disease Center, Rush University Medical Center, Chicago, Illinois (Bennett): Department of Neurology, Mayo Clinic, Rochester, Minnesota (Boeve); Hope Center for Neurological Disorders, Washington University, St Louis, Missouri (Cruchaga); Department of Medical and Molecular Genetics, Indiana University, Indianapolis (Faber Foroud); Department of Neurology, Indiana University Center for Alzheimer's Disease and Related Disorders, Indianapolis (Farlow) Department of Neuroscience, Mount Sinai School of Medicine, New York, New York (Goate); Department of Neurology, Mayo Clinic, Jacksonville, Florida (Graff-Radford); Department of Medicine, Columbia University, New York, New York (Lantigua); Department of Epidemiology, Columbia University, New York, New York (Ottman, Schupf, Mayeux); Department of Neurology and Neurotherapeutics, University of Texas Southwestern Medical Center, Dallas (Rosenberg); Editor, JAMA Neurology (Rosenberg); Department of Health Sciences Research, Mayo Clinic, Rochester, Minnesota (Schaid); Department of Psychiatry, University of Pittsburgh, Pittsburgh, Pennsylvania (Sweet); Department of Neurology, University of Pittsburgh, Pittsburgh, Pennsylvania (Sweet); Department of Epidemiology, University of Pittsburgh, Pittsburgh, Pennsylvania (Sweet).

Author Contributions: Dr Mayeux had full access to all the data in the study and takes responsibility for the integrity of the data and the accuracy of the data analysis.

Study concept and design: Tosto, Ottman, Schupf, Sweet, Mayeux.

Acquisition, analysis, or interpretation of data: All authors.

Drafting of the manuscript: Tosto, Mayeux. Critical revision of the manuscript for important intellectual content: All authors.

Statistical analysis: Tosto, Mayeux.

Obtained funding: Bennett, Brickman, Foroud,

Ottman, Sweet, Mayeux.

Administrative, technical, or material support
Bennett, Faber, Foroud, Farlow, Goate Graff-Radford, Lantigua, Mayeux. Study supervision: Tosto, Mayeux.

Conflict of Interest Disclosures: Dr Boeve reports serving as an investigator for clinical trials sponsored by Cephalon, Inc, Allon Pharmaceuticals, and GE Healthcare; receiving royalties from the publication of a book titled Behavioral Neurology of Dementia (Cambridge Medicine; 2009); receiving honoraria from the American Academy of Neurology; serving on the Scientific Advisory Board of the Tau Consortium; and receiving research support from the National Institute on Aging (NIA) (grants P50 AG016574, U01 AG006786, RO1 AG032306, RO1 AG041797) and the Mangurian Foundation. Dr Farlow reports receiving grant and research support from Accera, Biogen, Eisai Med Res, Eli Lilly \& Company, Genentech, MedAvante/ AstraZeneca, and Navidea; serving on the speaker's bureau at Eisai Med Res, Pfizer, Inc, Forest, Novartis, and Eli Lilly \& Company; serving on the consultant or advisory boards at Accera, Alltech, Avanir, Eisai Med Res, Inc, Helicon, Medavante, Medivation, Inc, Merck and Co, Inc, Novartis, Pfizer, Inc, Prana Biotech, QR Pharma, Roche, SanofiAventis, Schering-Plough, Toyama Pharm, Eli Lilly \& Company, UCB Pharma, and Elan. Dr Rosenberg reports holding a US patent for Amyloid $\beta$ Gene Vaccines and serving on the editorial board of the Journal of the Neurological Sciences. No other disclosures were reported.

Funding/Support: This study was supported by grants R01AG041797, U24AG026395, R01AG037212, P01AG007232, and U24AG21886 from the NIA.

Role of the Sponsor: The funding source had no role in the design and conduct of the study; collection, management, analysis, or interpretation of the data; preparation, review, or approval of the manuscript; and decision to submit the manuscript for publication.

Group Information: Members of the National Institute on Aging Late-Onset Alzheimer Disease/ National Cell Repository for Alzheimer Disease (NIA-LOAD NCRAD) Family Study Group include the following: Richard Mayeux, MD, MSc; Martin Farlow, MD; Tatiana Foroud, PhD; Kelley Faber, MS; Bradley F. Boeve, MD; Neill R. Graff-Radford, MD; David A Bennett, MD; Robert A. Sweet, MD; Roger Rosenberg, MD; Thomas D. Bird, MD; Carlos Cruchaga, PhD; and Jeremy M. Silverman, PhD.

Disclaimer: Dr Rosenberg is the editor of JAMA Neurology and serves on the editorial board of $J A M A$. He was not involved in the editorial evaluation or decision to accept this article for publication.

\section{REFERENCES}

1. Citron M. Alzheimer's disease: strategies for disease modification. Nat Rev Drug Discov. 2010;9 (5):387-398.
2. Cheng D, Noble J, Tang MX, Schupf N, Mayeux R, Luchsinger JA. Type 2 diabetes and late-onset Alzheimer's disease. Dement Geriatr Cogn Disord. 2011;31(6):424-430.

3. Luchsinger JA, Tang M-X, Stern Y, Shea S, Mayeux R. Diabetes mellitus and risk of Alzheimer's disease and dementia with stroke in a multiethnic cohort. Am J Epidemiol. 2001;154(7):635-641.

4. Luchsinger JA, Reitz C, Honig LS, Tang MX, Shea $S$. Mayeux R. Aggregation of vascular risk factors and risk of incident Alzheimer disease. Neurology. 2005;65(4):545-551.

5. Reitz C, Tang M-X, Schupf N, Manly JJ, Mayeux R, Luchsinger JA. Association of higher levels of high-density lipoprotein cholesterol in elderly individuals and lower risk of late-onset Alzheimer disease. Arch Neurol. 2010;67(12):1491-1497.

6. Reitz C, Tang M-X, Schupf N, Manly JJ, Mayeux $R$, Luchsinger JA. A summary risk score for the prediction of Alzheimer disease in elderly persons. Arch Neurol. 2010;67(7):835-841.

7. Bangen KJ, Nation DA, Clark LR, et al. Interactive effects of vascular risk burden and advanced age on cerebral blood flow. Front Aging Neurosci. 2014;6:159.

8. Kalaria RN. Vascular basis for brain degeneration: faltering controls and risk factors for dementia. Nutr Rev. 2010;68(suppl 2):S74-S87.

9. Chui HC, Zheng L, Reed BR, Vinters HV, Mack WJ. Vascular risk factors and Alzheimer's disease: are these risk factors for plaques and tangles or for concomitant vascular pathology that increases the likelihood of dementia? an evidence-based review. Alzheimers Res Ther. 2012;4(1):1.

10. Toledo JB, Arnold SE, Raible K, et al. Contribution of cerebrovascular disease in autopsy confirmed neurodegenerative disease cases in the National Alzheimer's Coordinating Centre. Brain. 2013;136(pt 9):2697-2706.

11. Yarchoan $M$, Xie SX, Kling MA, et al. Cerebrovascular atherosclerosis correlates with Alzheimer pathology in neurodegenerative dementias. Brain. 2012;135(pt 12):3749-3756.

12. Honig LS, Tang M-X, Albert $S$, et al. Stroke and the risk of Alzheimer disease. Arch Neurol. 2003;60 (12):1707-1712.

13. Attems J, Jellinger KA. The overlap between vascular disease and Alzheimer's disease: lessons from pathology. BMC Med. 2014;12(1):206.

14. Vardarajan BN, Faber KM, Bird TD, et al; NIA-LOAD/NCRAD Family Study Group. Age-specific incidence rates for dementia and Alzheimer disease in NIA-LOAD/NCRAD and EFIGA families: National Institute on Aging Genetics Initiative for Late-Onset Alzheimer Disease/National Cell Repository for Alzheimer Disease (NIA-LOAD/NCRAD) and Estudio Familiar de Influencia Genetica en Alzheimer (EFIGA). JAMA Neurol. 2014;71(3):315-323. 
15. McKhann G, Drachman D, Folstein M, Katzman R, Price D, Stadlan EM. Clinical diagnosis of Alzheimer's disease: report of the NINCDS-ADRDA Work Group under the auspices of Department of Health and Human Services Task Force on Alzheimer's Disease. Neurology. 1984;34 (7):939-944.

16. Tosto G, Fu H, Vardarajan BN, et al. F-box/LRR-repeat protein 7 is genetically associated with Alzheimer's disease. Ann Clin Transl Neurol. 2015:2(8):810-820.

17. Chobanian AV, Bakris GL, Black HR, et al; Joint National Committee on Prevention, Detection, Evaluation, and Treatment of High Blood Pressure. National Heart, Lung, and Blood Institute; National High Blood Pressure Education Program Coordinating Committee. The seventh report of the Joint National Committee on prevention, detection, evaluation, and treatment of high blood pressure. Hypertension. 2003;42(6):1206-1252.

18. Aho K, Harmsen P, Hatano S, Marquardsen J, Smirnov VE, Strasser T. Cerebrovascular disease in the community: results of a WHO collaborative study. Bull World Health Organ. 1980;58(1):113-130.

19. Lambert JC, Ibrahim-Verbaas CA, Harold D, et al; European Alzheimer's Disease Initiative (EADI); Genetic and Environmental Risk in Alzheimer's Disease; Alzheimer's Disease Genetic Consortium; Cohorts for Heart and Aging Research in Genomic Epidemiology. Meta-analysis of 74,046 individuals identifies 11 new susceptibility loci for Alzheimer's disease. Nat Genet. 2013;45(12): 1452-1458.

20. Wijsman EM, Pankratz ND, Choi Y, et al: NIA-LOAD/NCRAD Family Study Group. Genome-wide association of familial late-onset Alzheimer's disease replicates BIN1 and CLU and nominates CUGBP2 in interaction with $A P O E$. PLOS Genet. 2011;7(2):e1001308.

21. Nelder JA, Mead R. A simplex method for function minimization. Comput J. 1965;7(4):308-313.

22. Preacher KJ, Hayes AF. Asymptotic and resampling strategies for assessing and comparing indirect effects in multiple mediator models. Behav Res Methods. 2008:40(3):879-891.
23. Sobel ME. Asymptotic confidence intervals for indirect effects in structural equation models. Sociol Methodol. 1982:13:290-312.

24. Djoussé $L$, Myers RH, Province MA, et al. Influence of apolipoprotein E, smoking, and alcoho intake on carotid atherosclerosis: National Heart,

Lung, and Blood Institute Family Heart Study. Stroke. 2002;33(5):1357-1361.

25. Østergaard SD, Mukherjee S, Sharp SJ, et al; Alzheimer's Disease Genetics Consortium; GERAD1 Consortium; EPIC-InterAct Consortium. Associations between potentially modifiable risk factors and Alzheimer disease: a mendelian randomization study. PLoS Med. 2015;12(6):e1001841.

26. Guo Z, Viitanen M, Fratiglioni L, Winblad B. Low blood pressure and dementia in elderly people: the Kungsholmen project. BMJ. 1996;312(7034):805-808.

27. Albanese E, Lombardo FL, Prince MJ, Stewart R. Dementia and lower blood pressure in Latin America, India, and China: a 10/66 cross-cohort study. Neurology. 2013;81(3):228-235.

28. Chuang Y-F, Breitner JC, Chiu Y-L, et al; Cache County Investigators. Use of diuretics is associated with reduced risk of Alzheimer's disease: the Cache County Study. Neurobiol Aging. 2014;35(11):2429-2435.

29. Gelber RP, Ross GW, Petrovitch H, Masaki KH, Launer LJ, White LR. Antihypertensive medication use and risk of cognitive impairment: the Honolulu-Asia Aging Study. Neurology. 2013;81(10) 888-895.

30. Nimmrich V, Eckert A. Calcium channel blockers and dementia. Br J Pharmacol. 2013;169(6):1203-1210.

31. Mogi M, Horiuchi M. Effects of angiotensin II receptor blockers on dementia. Hypertens Res. 2009;32(9):738-740.

32. Yasar S, Xia J, Yao W, et al; Ginkgo Evaluation of Memory (GEM) Study Investigators. Antihypertensive drugs decrease risk of Alzheimer disease: Ginkgo Evaluation of Memory Study. Neurology. 2013;81(10):896-903.

33. Qiu WQ, Mwamburi M, Besser LM, et al. Angiotensin converting enzyme inhibitors and the reduced risk of Alzheimer's disease in the absence of apolipoprotein E4 allele. J Alzheimers Dis. 2013;37(2):421-428.
34. Imfeld $\mathrm{P}$, Bodmer $\mathrm{M}$, Schuerch $\mathrm{M}$, Jick SS, Meier CR. Risk of incident stroke in patients with Alzheimer disease or vascular dementia. Neurology. 2013:81(10):910-919.

35. Hayes AF, Preacher KJ. Quantifying and testing indirect effects in simple mediation models when the constituent paths are nonlinear. Multivariate Behav Res. 2010;45(4):627-660.

36. Tosto G, Zimmerman ME, Carmichael OT, Brickman AM; Alzheimer's Disease Neuroimaging Initiative. Predicting aggressive decline in mild cognitive impairment: the importance of white matter hyperintensities. JAMA Neurol. 2014;71(7): $872-877$.

37. Tosto G, Zimmerman ME, Hamilton JL, Carmichael OT, Brickman AM; Alzheimer's Disease Neuroimaging Initiative. The effect of white matter hyperintensities on neurodegeneration in mild cognitive impairment. Alzheimers Dement. 2015;11 (12):1510-1519.

38. Li X, Song D, Leng SX. Link between type 2 diabetes and Alzheimer's disease: from epidemiology to mechanism and treatment. Clin Interv Aging. 2015;10:549-560.

39. Ahtiluoto $\mathrm{S}$, Polvikoski T, Peltonen $\mathrm{M}$, et al. Diabetes, Alzheimer disease, and vascular dementia: a population-based neuropathologic study. Neurology. 2010;75(13):1195-1202.

40. Peila R, Rodriguez BL, Launer LJ; Honolulu-Asia Aging Study. Type 2 diabetes, APOE gene, and the risk for dementia and related pathologies: the Honolulu-Asia Aging Study. Diabetes. 2002:51(4):1256-1262

41. Arvanitakis Z, Schneider JA, Wilson RS, et al. Diabetes is related to cerebral infarction but not to AD pathology in older persons. Neurology. 2006;67 (11):1960-1965.

42. Moran C, Beare R, Phan TG, Bruce DG, Callisaya ML, Srikanth V; Alzheimer's Disease Neuroimaging Initiative (ADNI). Type 2 diabetes mellitus and biomarkers of neurodegeneration. Neurology. 2015;85(13):1123-1130. 